\title{
Constitutive expression of heat shock protein p23 correlates with proneural territories in imaginal discs of Drosophila melanogaster
}

\author{
Jana Alonso ${ }^{1, *}$, Javier M. Rodriguez, ${ }^{1,}$, Luis Alberto Baena-López ${ }^{1, *}$, \\ Maria Teresa Alonso ${ }^{2}$ and Juan F. Santarén ${ }^{1}$ \\ ${ }^{1}$ Centro de Biología Molecular "Severo Ochoa", CSIC-UAM, Universidad Autónoma de Madrid, \\ Cantoblanco, Madrid, Spain \\ ${ }^{2}$ SIDI, Universidad Autónoma de Madrid, Cantoblanco, Madrid, Spain
}

2-DE followed by MALDI-TOF was used to purify and identify a Drosophila protein (catalogued as SSP 6002) that showed marked differences in the level of expression in the different imaginal discs of third instar larvae. Fingerprinting showed that the spot of interest was the heat shock 23 polypeptide (hsp23). We characterized the kinetics of its induction by heat shock in wing imaginal discs and raised an antiserum against the denatured protein, which recognizes a single unphosphorylated spot on 2-D gels. The difference in its expression in discs was corroborated by analyzing its level in the imaginal discs of postbithorax mutants. We also investigated the developmental expression of hsp23 in imaginal discs with antiserum raised against the native protein. Its spatial and temporal pattern of expression is related to the proneural territories and maintained even under heat shock conditions. In addition, its pattern of expression is regulated by transcription factors and signaling pathways (notch and epidermal growth factor receptor) involved in proneural specification.

\section{Keywords:}

Drosophila / Heat shock / hsp23 / Imaginal discs / Proneural differentiation
Received: October 19, 2004 Revised: December 15, 2004 Accepted: December 27, 2004

\section{Introduction}

We use high-resolution 2-DE to analyze aspects of the development, morphogenesis, and genetic variation of the wing imaginal discs of mature larvae of Drosophila melanogaster as a model system. For this purpose, we constructed a protein database that was used in a previous study to compare the

Correspondence: Dr. Juan F. Santarén, Centro de Biología Molecular "Severo Ochoa", Facultad de Ciencias, Universidad Autónoma, Cantoblanco, 28049 Madrid, Spain

E-mail: jfsantaren@cbm.uam.es

Fax: +34-91-4974799

\begin{abstract}
Abbreviations: shsps, small heat shock proteins; hsp23, heat shock 23 polypeptide; apG4, apterous-G4; dppG4, decapentaplegic-G4 A.3; UAS-sc, UAS-scute; UAS- $\boldsymbol{N}_{i}$ UAS-Notch ${ }_{\text {intra; }} \mathbf{w g}$, wingless; AC-C, achaete-scute complex; sc, scute; bs, blistered; EGFR, notch (N) and epidermal growth factor receptor; SMCs, sensory organ mother cells
\end{abstract}

standard wing pattern with those of haltere, leg 1, leg 2, leg 3, and eye-antenna imaginal discs of the same developmental stage. We thus defined a set of 17 polypeptides whose levels of expression varied between the different imaginal discs [1]. In the present study, we tried to purify and identify one of those proteins, specifically an acidic protein that was named IEF 41 in the original report and later catalogued as SSP 6002 when computerized quantitative analysis was introduced [2]. By preparative 2-DE and MALDI-TOF, we identified the protein as hsp23, one of the four small heat shock proteins of Drosophila (hsp22, hsp23, hsp26, and hsp27), whose genes cluster within $12 \mathrm{~kb}$ of DNA at cytological locus 67B. This locus encodes three other genes that were first identified by their expression at specific stages of development [3] and whose transcripts are also activated after heat shock. The beginning of the pupal period is the only stage of D. melanogaster development during which these

\footnotetext{
* These authors contributed equally.
} 
seven heat shock genes are expressed [4]. Nevertheless, the levels of their transcripts differ widely, ranging from high levels of the hsp23 message, which is as abundant as the mRNA for cytoplasmic actin, to the roughly 100 -fold lower levels of the hsp22 transcript [5]. Although the small heat shock proteins (shsps) represent the least conserved subfamily of heat shock proteins, comparison of their predicted amino acid sequences has revealed a highly conserved region of 108 amino acid residues representing 51 to $62 \%$ of their lengths [6].

Studies in different experimental systems have revealed a variety of functions for shsps under stress conditions. These include basic chaperone activity [7], cytoskeleton protection [8], and modulation of the apoptotic process [9], all of which are aspects of cellular defense against environmental stresses. The physiological role of these proteins remains largely a matter of conjecture, although it is known that during a heat shock they are found mainly in the nucleus. During the recovery period at $25^{\circ} \mathrm{C}$ following a heat shock, they tend to redistribute between the nucleus and the cytoplasm, where they appear to aggregate into high molecular weight 20-30S cytoplasmic complexes associated with RNA [10].

In contrast to the classical definition of heat shock proteins induced by stress, cell-specific expression of shsps in the absence of stress has been reported during the development of a wide variety of organisms including Caenorhabditis elegans [11], Drosophila melanogaster [12], Xenopus laevis [13], Mus musculus [14], and man [15]. Their characteristic cellspecific pattern of expression has led to the idea that they may be involved in aspects of differentiation. For example, during normal Drosophila development, the shsps are under differential control and only a subset are present at certain stages. Thus, expression of hsp26 and hsp27 follows a tissuespecific pattern [16]. Equally, during oogenesis, hsp27 has a stage-specific localization within nurse and follicle cells [17], while hsp23, hsp26, and hsp27 are expressed in different cell types during spermatogenesis [18]. During embryogenesis, hsp27 is associated with cells of the brain and of the ventral nerve cord, while hsp26 is found exclusively in the gonads [19].

Hsp23 has also been described as displaying a cell-specific pattern of expression during embryonic neurogenesis [20], and has recently been shown to be strongly down-regulated following targeted expression of the glial master gene, $\mathrm{gcm}$ [21]. Its expression in the neuronal and glial lineage of the CNS demonstrates that its expression in the neuroectoderm is closely and autonomously linked to the acquisition of MP2 fate, as it does not require the presence of a functional midline [22]. In vitro transactivation assays indicate that the Single-minded, Tango, and Drifter transcription factors, which are all involved in midline determination and differentiation, also regulate $h s p 23$ promotor activity. Loss of hsp23 appears not to be detrimental to CNS formation.

Information concerning the expression and distribution of these shsps could be useful for correlating their sites of action with possible functions. In this work, we report the characterization, expression, and location of hsp23 in nonheat-shocked Drosophila imaginal discs, using a polyclonal antibody raised against the native protein. We show that its expression varies temporally in a manner dependent on the particular imaginal disc, and, more importantly, seems to be independent of temperature. Furthermore, it is cell-specific and correlates with proneural territories in the various imaginal discs, being regulated by proneural transcription factors and signaling pathways notch $(\mathrm{N})$ and epidermal growth factor receptor (EGFR).

\section{Materials and methods}

\subsection{Fly strains and cell culture}

We used a laboratory stock of wild-type D. melanogaster (strain Vallecas (Spain)). The GAL 4 strains used to drive UAS-transgenes were apterous-G4 (apG4) [23] and decapentaplegic-G4 A.3 (dppG4) [24]. The UAS-transgene strains used were UAS-scute (UAS-sc) [25], UAS-Notch intra $\left(U A S-N_{i}\right)$

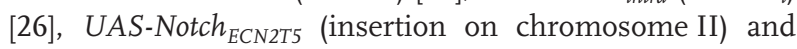

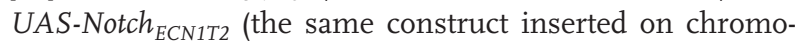
some III) (UAS-N $N_{E C N}$ ) [27], UAS-RasV12 (UAS-RasV12) [28], and UAS-Raf dominant negative 3.1 (UAS-Raf3.1 ${ }_{D N}$ ) [29]. All overexpression experiments were carried out at $25^{\circ} \mathrm{C}$. The neuralized (neur)-lacZ A101.IF3 strain that reproduces the wild-type expression pattern of neuralized [30] was used as a marker of neuronal differentiation. The mutant $\mathrm{pbx}^{1} / \mathrm{Ubx}^{109}$ was obtained from our stock collection.

The established cell line used was derived from wing imaginal discs of mature larvae of $D$. melanogaster (strain Oregon R), and designated CME W2. Cells $\left(3 \times 10^{5}\right)$ were plated in multiwell plates $\left(2 \mathrm{~cm}^{2}\right)$ as described elsewhere [31].

\subsection{Preparation of samples and 2-DE}

Dissection and labeling of imaginal discs were carried out as described previously [32]. Analytical 2-DE was performed according to O'Farrell [33] with some modifications [32]. Second-dimension separations were carried out on $15 \%$ polyacrylamide gels $(24 \times 24 \mathrm{~cm})$ and run overnight at room temperature $\left(18^{\circ} \mathrm{C}\right)$. Gels were processed for fluorography [34], dried, and exposed at $-70^{\circ} \mathrm{C}$ for various periods of time. For preparative gels, the cells from 20 confluent $65 \mathrm{~cm}^{2}$ plates of CME W2 cells were used to prepare proteins as previously described [31].

\subsection{Computer analysis of 2-D patterns}

Four fluorograms, each after different exposure times $(4,24$, 96 and $240 \mathrm{~h}$ ), were made from each gel and digitized at $176 \times 176 \mu \mathrm{m}$ resolution with a pdi scanning densitometer 
(Bio-Rad). The resulting 2-D images were merged and analyzed with PDQuest software (version 5.0) running on a SPARC station IPC microcomputer (Sun Microsystems).

\subsection{In-gel digestion and MALDI-TOF analysis}

Coomassie protein spots were excised from the gel, digested with trypsin and processed as described elsewhere [35]. The peptide solutions were analyzed with the Applied Biosystems 4700 proteomics analyzer (Applied Biosystems, Framingham, MA, USA) with TOF/TOF ion optics. MS spectra were obtained in reflectron mode using an acceleration voltage of $1 \mathrm{kV}$. Desorption and ionization of samples were performed with an Nd:YAG operating at $355 \mathrm{~nm}$. The final mass spectra was produced by averaging 3600 laser shots. The autodigestion products of trypsin were used for internal calibration. MS/MS sequencing analysis was performed with the same equipment.

\subsection{Protein identification using the Drosophila database}

Protein was identified by mass fingerprinting. The monoisotopic PMF data obtained by MALDI-TOF were used to search the Swiss-Prot/TrEMBL non-redundant protein database.

\subsection{Antibodies against Hsp23}

We made two antibodies, one $\left(\alpha-\mathrm{hsp} 23_{\text {Den }}\right)$ against denaturated polypeptide, the other $\left(\alpha-h s p 23_{N a t}\right)$ against native hsp23. In the former case, the cell contents of 14 confluent $500-\mathrm{cm}^{2}$ trays (Nunc) of CME W2 cells were used to prepare the SSP 6002 polypeptide for immunization. The cell monolayers were washed with Hanks' solution, and scraped off with a rubber policeman in $10 \mathrm{~mL}$ of the same. After centrifugation, the cells were sonicated, treated with DNase and RNase, and passed several times through a narrow-gauge needle. The sample was then lyophilized and resuspended in $5 \mathrm{~mL}$ of lysis buffer, after which $\left[{ }^{35} \mathrm{~S}\right]$ methionine $+\left[{ }^{35} \mathrm{~S}\right] \mathrm{cys}-$ teine-labeled proteins from wing imaginal discs were added to identify the polypeptides by autoradiography. One hundred 2-D gels were run as described above, immediately dried (without fixation), and exposed for 4 days. The hsp23 protein was located via the autoradiographs and cut out of the gels. The gel pieces were rehydrated and the protein recovered by electroelution. Approximately $150 \mu \mathrm{g}$ of this protein was injected into a New Zealand white rabbit, and rabbit sera were tested by immunoblotting against hsp23 as described in Section 2.7. To prepare the antibody against the native hsp23, a cDNA containing the complete ORF was cloned into the pRSET vector (Invitrogen, Leek, The Netherlands), and expressed as a $\mathrm{His}_{6}$-fusion protein in Escherichia coli BL21 (DE3)pLysS (Novagen, Madison, WI, USA). Approximately $240 \mu \mathrm{g}$ of this protein was purified by NiTA chromatography (Clontech, Palo Alto, CA, USA) and injected into two New Zealand white rabbits.

\subsection{Immunoblotting}

Proteins resolved by 2-D gels were transferred onto nitrocellulose filters at $130 \mathrm{~mA}$ for $6 \mathrm{~h}$, and the filters were incubated for $120 \mathrm{~min}$ at room temperature in PBS containing $10 \%$ bovine serum and $\alpha$-hsp23 $3_{\text {Den }}$ diluted to 1:100. The filters were then incubated with peroxidase-conjugated antirabbit immunoglobulin G (IgG) for $120 \mathrm{~min}$ in the same buffer and immunocomplexes visualized with diaminobenzidine as substrate.

\subsection{Immunostaining of embryos and imaginal discs}

Standard procedures were used for whole-mount immunohistochemistry [36]. Imaginal discs were stained as described previously [37]. The $\alpha-\mathrm{Hsp} 23_{\mathrm{Nat}}$ was used at a 1:2000 dilution in all experiments. Mouse anti-Blistered (provided by M. Affolter), mouse anti-Wingless (Hybridoma Bank), and

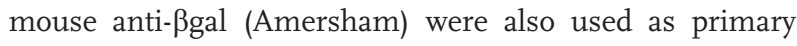
antibodies. Alexa Fluor-488, Fluor-546 (Molecular Probes), and Cy5 (Jackson Inmunoresearch) were used to detect the primary antibodies.

\subsection{Microscopy and image treatment}

Imaginal discs were mounted on Vectashield (Vector Laboratories). Images were acquired with a Bio-Rad 2000 confocal microscope and treated with Metaview (Universal Imaging) and Photoshop 7.0 (Adobe) software. To avoid focal problems, we captured and merged at least 15 planes of the various imaginal discs.

\section{Results}

\subsection{Expression of polypeptide SSP 6002 varies in different imaginal discs}

Figure 1A shows a representative fluorogram of a 2-D gel of the acidic polypeptides from wing imaginal discs of mature larvae (wild type, strain Vallecas). The gels were analyzed on a computer with the PDQuest system and each polypeptide was assigned a number in the database. In this way we compiled a database of $1492\left[{ }^{35} \mathrm{~S}\right]$ methionine $+\left[{ }^{35} \mathrm{~S}\right]$ cysteinelabeled polypeptides (1226acidic, IEF and 266 basic, NEPHGE). In a previous paper [1], we compared the patterns of protein synthesis in six different imaginal discs. We observed some quantitative differences in 17 polypeptides, one of which was catalogued as IEF 41 in our "manual" database [32], and corresponds to SSP 6002 in the catalogue obtained by computer analysis [2]. In the present study, we quantified the level of expression of this polypeptide in various imaginal discs. To do that, wing, haltere, leg 1 , leg 2 , leg 3, and eye-antenna imaginal discs were labeled with $\left[{ }^{35} \mathrm{~S}\right]$ methionine $+\left[{ }^{35} \mathrm{~S}\right]$ cysteine, resolved on 2-D gels, and quantified with the PDQuest software. Figure 1B shows a 

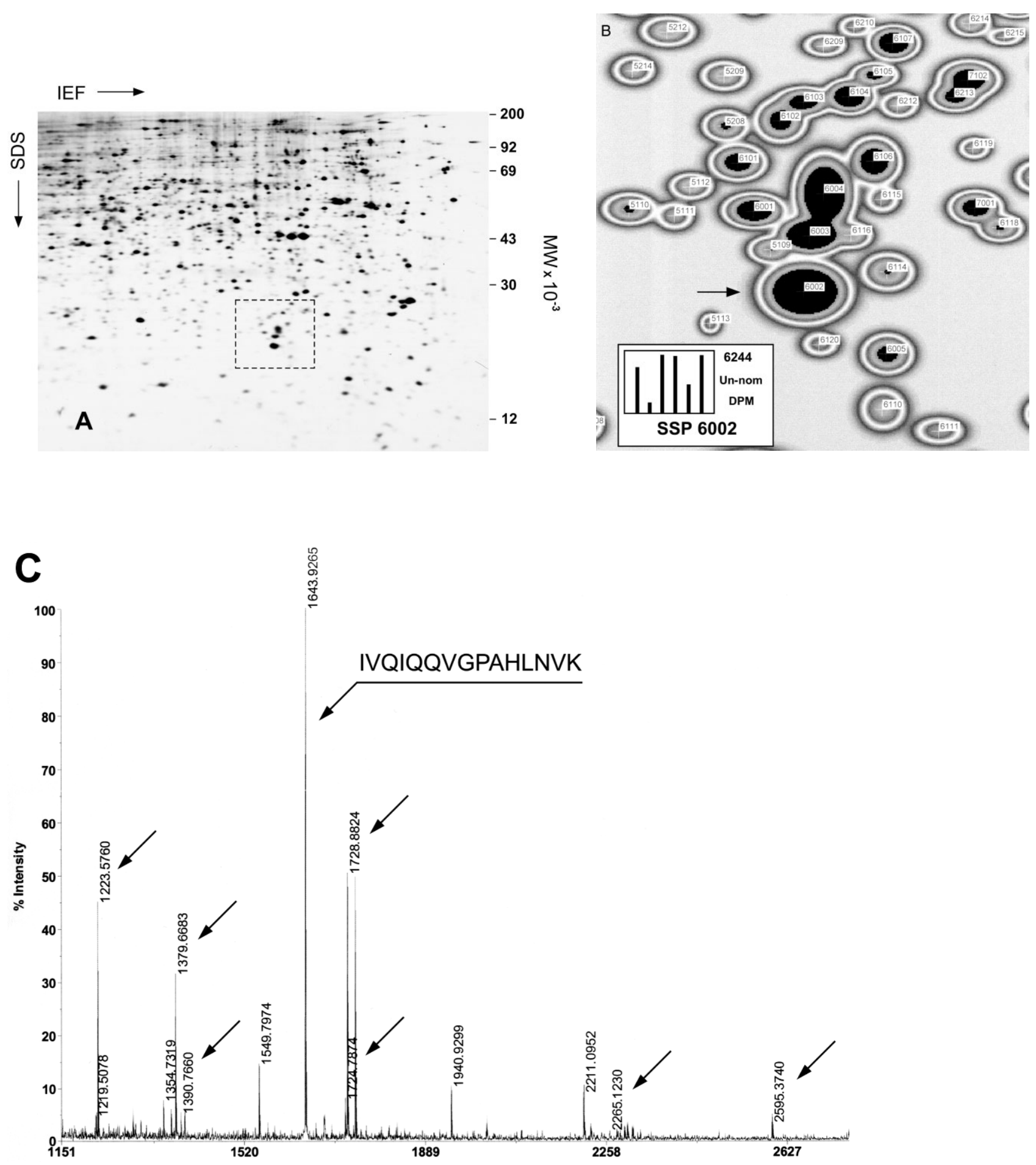

Figure 1. 2-DE (IEF) of total $\left[{ }^{35} \mathrm{~S}\right]$ methionine $+\left[{ }^{35} \mathrm{~S}\right]$ cysteine-labeled polypeptides in wing imaginal discs of late third instar larvae of Drosophila melanogaster. (A) The $\mathrm{pH}$ ranges from 7.0 (left) to 4.5 (right). The area shown in (B) is boxed. (B) Close-up of the boxed region in (A); polypeptide SSP 6002 is indicated by the arrow. Inset: each bar represents the quantification of the SSP 6002 polypeptide in each gel of the matchset; from left to right the bars represent the wing, haltere, leg 1 , leg 2 , leg 3 , and eye-antenna imaginal discs. The number in the upper right of the histogram is the quantification of the maximum bar in the graph. The other bars are drawn in proportion to the highest bar. (C) MALDI-TOF spectrum of a tryptic digest of SSP 6002. Peaks with arrows represent the peptides matched to $D$. melanogaster hsp23. The MS/MS spectrum of the 1643.92 peptide corresponds to the sequence IVQIQQVGPAHLNVK. 
detail of the IEF gel of wing imaginal discs containing this polypeptide and the quantification of its level of expression in the six imaginal discs. Taking the wing imaginal discs as reference, the level of the polypeptide was higher in leg 1 , leg 2, and eye-antenna and lower in haltere and leg 3. We therefore focused on the identification and characterization of SSP 6002.

\subsection{Identification of SSP 6002 polypeptide as hsp23}

In order to identify SSP 6002, we isolated 100 wing imaginal discs and lyophilized and resuspended them in $40 \mu \mathrm{L}$ of lysis buffer. To the lysate we added $10^{6} \mathrm{cpm}$ of proteins from the wing imaginal discs labeled with $\left[{ }^{35} \mathrm{~S}\right]$ methionine $+\left[{ }^{35} \mathrm{~S}\right]$ cysteine and we ran a 2-D gel with this mixture. The gel was stained with Coomassie, dried, and exposed to film for 4 days. The radiolabeled proteins acted as tracers, allowing the identification of polypeptide SSP 6002, which was located on the autoradiograph and later on the stained gel. A new 2-D gel, on this occasion obtained from only 100 wing imaginal discs, was run and stained with Coomassie, and the SSP 6002 spot was excised and processed for MALDI analysis. Figure $1 \mathrm{C}$ shows the MALDI-MS spectrum of the tryptic digest obtained. A database search identified SSP 6002 as heat shock protein 23 . The ten matching peptides covered $59 \%$ of the sequence of the protein. The assignment was corroborated by MALDI-TOF-TOF of ion 1643.93 whose sequence was determined to be IVQIQQVGPAHLNVK.

\subsection{Kinetics of heat shock induction of hsp23}

For heat shock experiments, ten wing imaginal discs were incubated at $25^{\circ} \mathrm{C}$ for $1 \mathrm{~h}$ (control, Fig. $2 \mathrm{~A}$ ), or at $37^{\circ} \mathrm{C}$ for $1 \mathrm{~h}$ or more. In all experiments, proteins were labeled with $\left[{ }^{35}\right.$ S $]$ methionine $+\left[{ }^{35} \mathrm{~S}\right]$ cysteine for the last hour of incubation, and analyzed by 2-DE and fluorography. Hsp23 was then identified by computerized gel scanning as described in Section 3.2. Figure 2B illustrates the significant changes visible on typical fluorograms after $2 \mathrm{~h}$ of incubation at $37^{\circ} \mathrm{C}$. With the heat shock the rate of synthesis of hsp23 increased, reaching a maximal increase of six-fold $2 \mathrm{~h}$ after heat stimulation. It then declined, reaching basal level at $5 \mathrm{~h}$ (Fig. 2C).

\subsection{Hsp23 is a unique and non-phosphorylated polypeptide}

In order to further characterize hsp23, we generated a polyclonal antibody against the denatured polypeptide. The specificity of the antibody was demonstrated by Western blotting of 2-D gels. Forty wing imaginal discs were labeled as described, separated on 2-D gels, transferred to a nitrocellulose filter, and exposed to antibody. The anti-hsp23 antibody recognized a single spot at the position of hsp23 (Fig. 3A), showing that hsp23 exists as an unmodified poly-
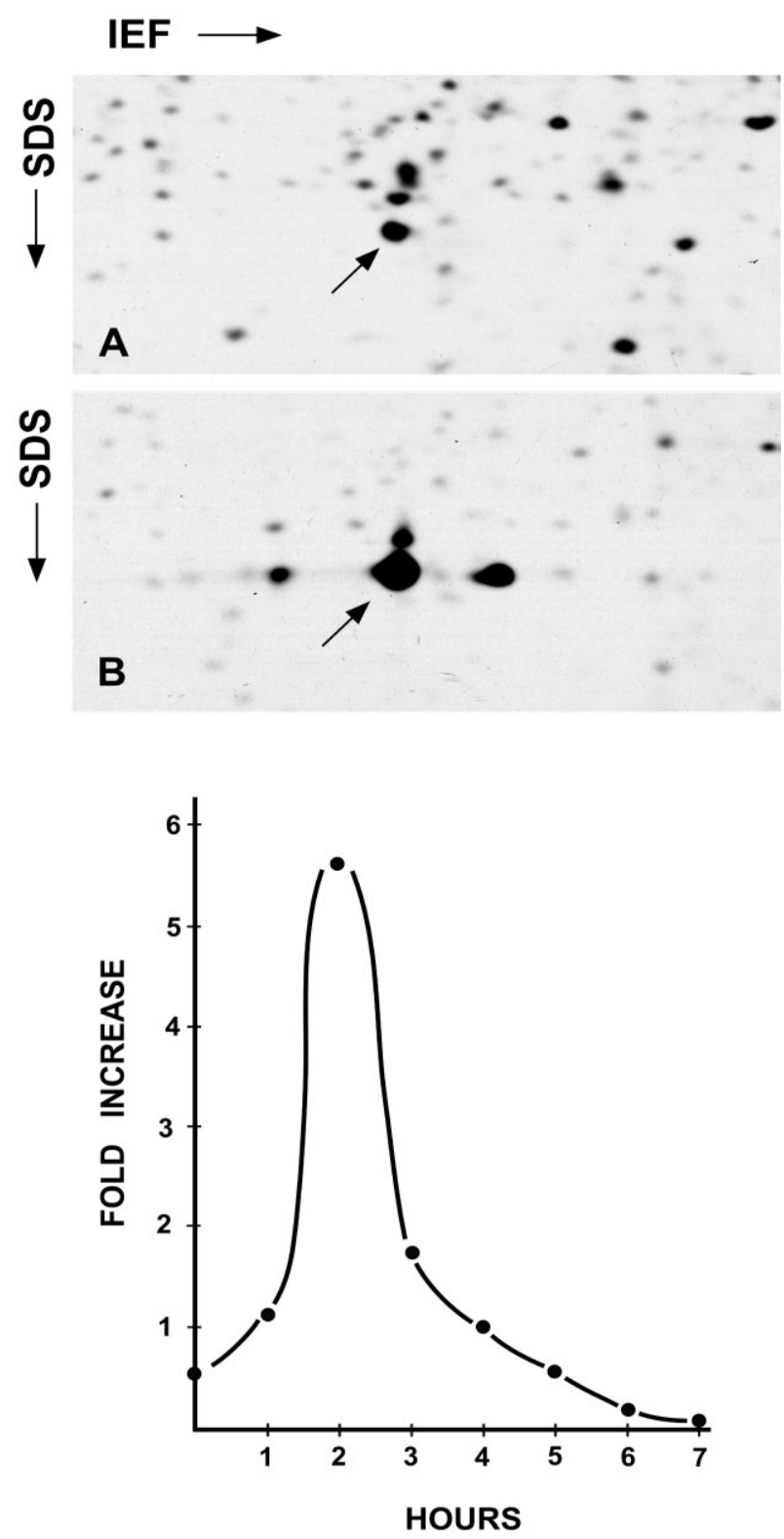

Figure 2. Close-up regions of 2-D gel separations (IEF) showing the effect of heat shock induction of hsp23 in wing imaginal discs of Drosophila. (A) Control after $60 \mathrm{~min}$ of labeling with $\left[{ }^{35} \mathrm{~S}\right]$ methionine $+\left[{ }^{35} \mathrm{~S}\right]$ cysteine at $25^{\circ} \mathrm{C}$ and $(\mathrm{B})$ induction after $120 \mathrm{~min}$ at $37^{\circ} \mathrm{C}$. (C) Time course of the induction of hsp23.

peptide. We carried out a similar experiment with $\left[{ }^{32} \mathrm{P}\right]$ labeling and did not observe any label in the hsp23 spot, showing that the polypeptide is not phosphorylated.

\subsection{Synthesis of hsp23 in $\mathrm{pbx}^{1} / \mathrm{Ubx}^{109}$ imaginal discs}

One of the greatest differences in the level of expression of hsp23 was between wing and haltere imaginal discs (Fig. 1B). In order to confirm this observation we compared the levels of expression of hsp23 in the wing and haltere 


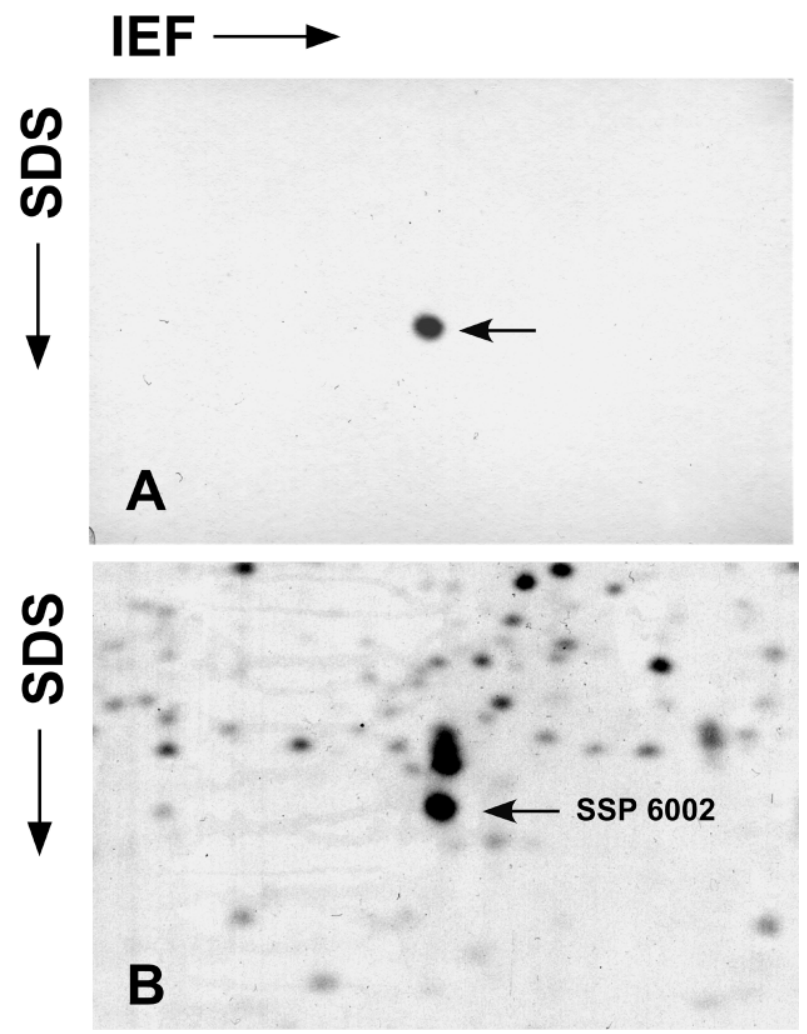

Figure 3. Specificity of the polyclonal antibody against hsp23 polypeptide. Twenty wing imaginal discs of late third instar larvae of $D$. melanogaster were labeled over $60 \mathrm{~min}$, resolved by 2-DE and transferred to nitrocellulose paper. The filter was analyzed with a polyclonal antibody against denatured SSP 6002. (B) Autoradiograph of (A).

imaginal disc of wild-type Drosophila flies with those observed in a postbithorax background. Flies homozygous for $\mathrm{pbx}^{1} / \mathrm{Ubx}^{109}$ have a homeotic transformation of halteres into wings, showing the classical "four wings" phenotype [38]. Wing and haltere imaginal discs from wild-type and $\mathrm{pbx}^{1} / \mathrm{Ubx}^{109}$ third instar larvae were dissected, labeled, and analyzed as before. As expected, hsp23 was present at similar levels in wild-type and mutant wing discs (Fig. 4A and C), whereas it was markedly elevated in the mutant haltere discs (Fig. 4D). These results corroborate and reinforce the differences observed previously between the different imaginal discs.

\subsection{Hsp23 has a defined and dynamic constitutive expression pattern during Drosophila development}

Because of the specificity of the data shown in the preceding sections, we decided to explore the idea that hsp23 does not merely act as a chaperone in imaginal discs. Hence, we analyzed its spatial and temporal pattern of expression in embryos and imaginal discs using a novel antibody against the native protein $\left(\alpha-h s p 23_{\text {Nat }}\right)$ (see Section 2$)$. Figure 5

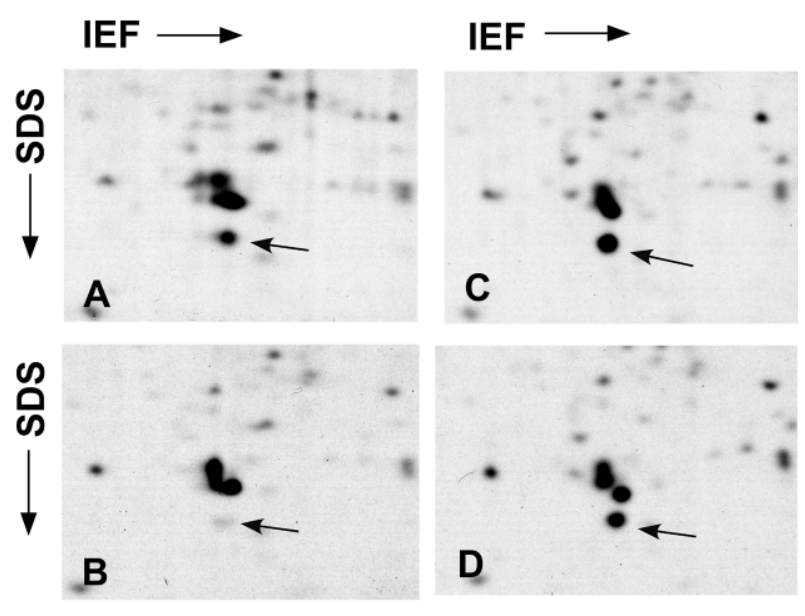

Figure 4. Close-ups of 2-D gels (IEF) of wild-type wing (A) and haltere $(B)$ imaginal discs, and the wing $(C)$ and haltere $(D)$ imaginal discs of a $\mathrm{pbx}^{1} / \mathrm{Ubx}^{109}$ mutant. Hsp23 is indicated by an arrow.

summarizes some of the results, in which the red channel always refers to hsp23. During embryonic development we detected similar profiles to those previously described [20]. Hsp23 was detected in the midgut (Fig. 5A) and in neurones within the extended germ band nervous system (Fig. 5B). The antibody also stained the nuclei of the salivary glands (Fig. 5C) and particular areas of the CNS (Fig. 5D) of third instar larvae. However, we focused our analysis on the wing, haltere, leg 1, leg 2, leg 3, and eye-antenna imaginal discs including wingless (wg) as a positional marker (green channel) in all the experiments. Wingless encodes a product with frizzled-2 binding activity involved in wing margin morphogenesis and is localized to the cytoplasm. After analyzing a substantial number of samples, some clear-cut conclusions emerged: first, that there was a defined temporally regulated pattern of expression in all cases; the faint and ill-defined expression in young imaginal discs being transformed into a well-defined pattern at the late third instar (red channel). Second, that the expression of hsp23 from the third medium instar onwards seemed to be restricted to proneural territories (proneural clusters) (Fig. 5E-F and H-K). Proneural clusters are defined by the precise spatio-temporal pattern of expression of one of several genes of the achaete-scute complex (AS-C) namely, achaete (ac), scute (sc), lethal of scute (l'sc), and asense (ase).

We extended the analysis of wing discs to the pupal period and included blistered (bs) as a marker (green channel). At that stage the antibody revealed a dynamic, restricted pattern of expression of hsp23 (red channel) that did not only coincide with proneural differentiation: a high level of the protein was present in the interveins during early pupal development and this later became restricted to the central domains of the interveins (Fig. 5G). This suggests that hsp23 has different roles at different stages of the development of imaginal discs. 

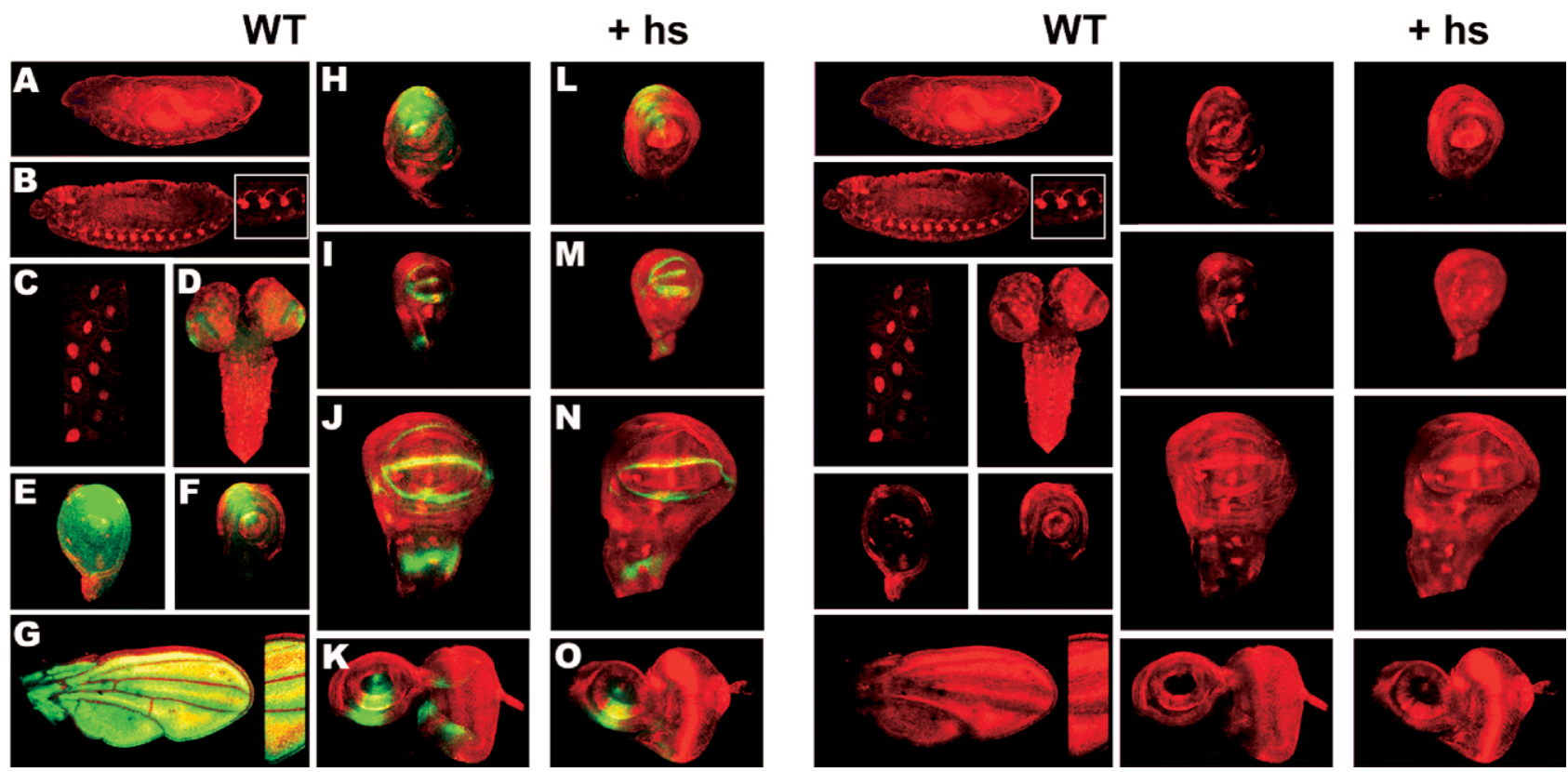

Figure 5. The pattern of Hsp23 is not modified by heat shock treatment. Protein expression pattern of hsp23 during Drosophila development in standard conditions $(A-K)$ and after heat shock (L-O). In all cases, expression of hsp23 is shown in the red channel while wg (A-F, $\mathrm{H}-\mathrm{K}$ ) or bs (G) is shown in the green channel. Hsp23 expression is visualized in the mesoderm (A) and nervous system (B) of the embryo (stage 13). Anterior and dorsal are to the left and top, respectively. Equally, hsp23 stains the nuclei in the salivary glands (C). Hsp23 has a restricted pattern in the CNS (D) and in different imaginal discs from third instar larvae as well as in pupal wings (G). In leg discs $1(\mathrm{E}), 2(\mathrm{~F})$, and $3(\mathrm{H})$ it is expressed in concentric rings and spots that are more intense ventrally (expression domain of $w g$ ). In the haltere (I), individual groups of cells express hsp23. In the wing, it is expressed in well-defined territories, such as the wing margin and groups of cells in the notum $(\mathrm{J})$. It is also present in the morphogenetic furrow of the eye and in the $w g$ domain of the antennae (K). Heat shock increases the levels of expression of hsp23 without altering its pattern (L-O).

Finally, to see whether the cell-specific pattern of expression was retained upon heat shock, third instar imaginal discs were heat-shocked for $2 \mathrm{~h}$ at $37^{\circ} \mathrm{C}$ to induce maximal synthesis of hsp23, and immediately fixed and analyzed with antibody. Surprisingly, although the level of hsp23 increased, its pattern was similar to that observed at $25^{\circ} \mathrm{C}$. This result was obtained with wing (Fig. $5 \mathrm{~N}$ ), haltere (Fig. $5 \mathrm{M}$ ), leg 3 (Fig. 5L), and eye-antenna (Fig. 5O) imaginal discs and suggests that although the amount of hsp23 synthesized is temperature-dependent, its pattern depends on epigenetic factors.

\subsection{Hsp23 is strictly regulated by several genetic factors and signaling pathways}

The restricted expression of hsp23 described in the previous section suggested that the protein was confined to proneural territories in mature imaginal discs. We therefore tried to demonstrate this by analyzing the co-expression of hsp23 and the neurogenic gene neuralized. Indeed, co-expression was clearly observed in all the cells of the proneural clusters, including the single mother cell (Fig. 6A). This result strongly suggests that expression of hsp23 is linked to proneural specification in all the imaginal discs.
An additional experiment was performed to see whether hsp23 could be regulated by proneural genes. To test this idea, we observed its expression in cells expressing sc ectopically in $d p p G 4$ territories. In contrast to the heat shock experiments, the $d p p$ domains of all the imaginal discs (eyes, legs, halteres, and wings) had a higher level of hsp23 expression, indicated with white arrowheads in Fig. 6B. This demonstrates that proneural genes regulate the expression of hsp23 and also suggests that this expression depends on the signaling pathways specifying the proneural cluster.

After the proneural clusters are specified, their size and integrity are controlled by the antagonistic actions of the $\mathrm{N}$ and EGFR signaling pathways [39]. We therefore drove ectopic expression of loss and gain functions of UAS-transgenes for these two signaling pathways with $d p p G 4$, and monitored expression of hsp23. This rose or fell in all the imaginal discs (eyes, legs, halteres, and wings) depending on loss or gain of $\mathrm{N}$ signaling (Fig. 6C and D). However, the EGFR pathway induced (white arrowheads) or repressed (yellow arrows) expression of hsp23 in a disc-dependent manner (Fig. 6E and F). Thus, the absence of EFGR signaling promoted ectopic expression of hsp23 in the wing and haltere while it repressed it in the leg (Fig. 6E). Strikingly, gain of function of EGFR signaling increased expression of hsp23 only in the proneural clusters of the wing and haltere imaginal discs, 

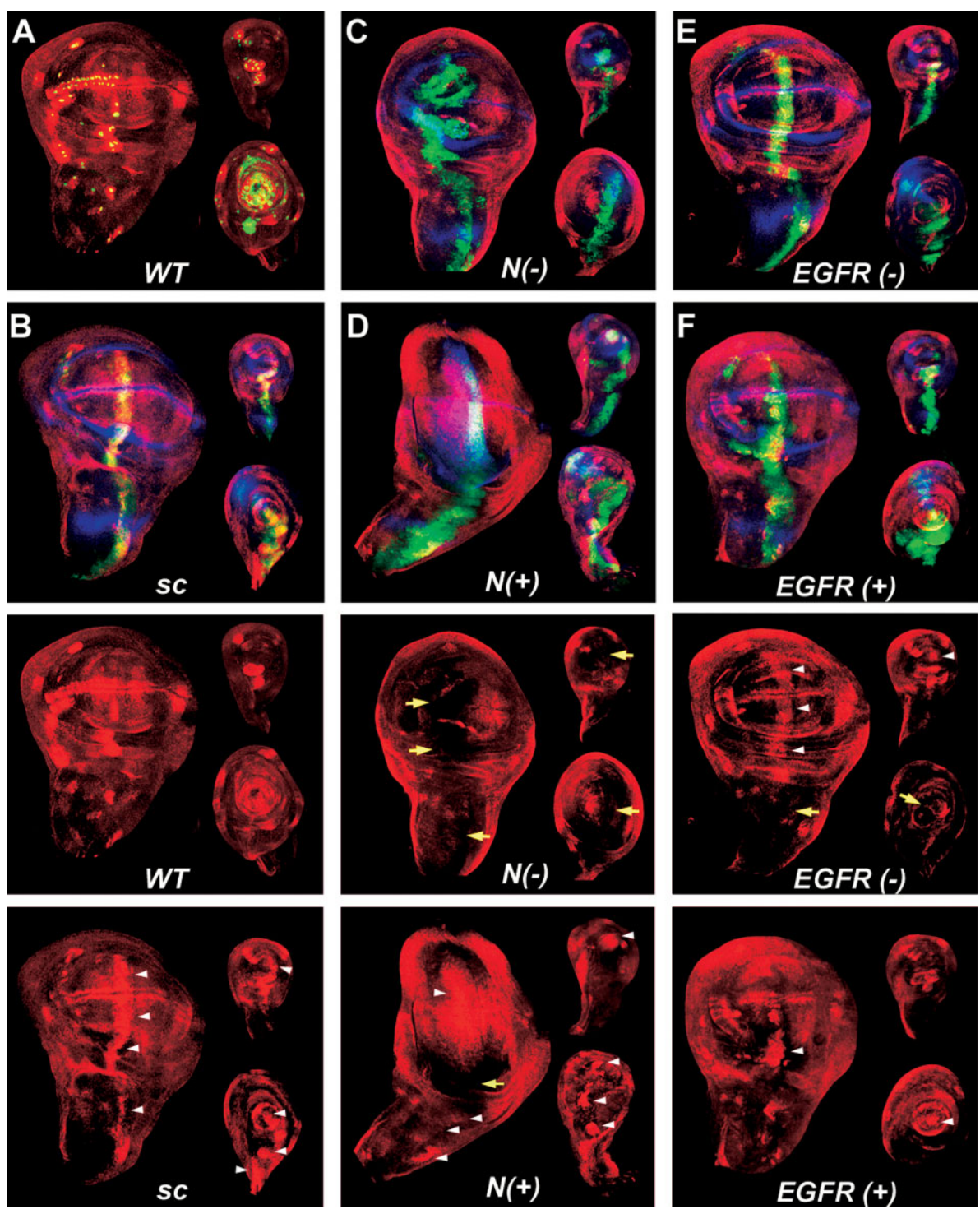

Figure 6. The expression pattern of hsp23 is dependent on transcription factors and signaling pathways involved in proneural specification. In all cases, expression of hsp23 is shown in the red channel and $w g$ in the blue channel. The green channel shows the expression of neuralized-lacz in (A) and defines the dppG4 domain of expression of the UAS-transgenes in (B-F). The imaginal discs monitored are: wing on the left, haltere on the upper right, and leg 3 on the lower right side of the panels. Ectopic expression of proneural genes (sc) in the dppG4 domain increases autonomously the level of hsp23 in each of the imaginal discs (B). Lack of $N$ signaling (overexpression of UAS- $\mathrm{N}_{\mathrm{ECN} 1 \mathrm{~T} 2}$ and UAS- $\mathrm{N}_{\mathrm{ECN} 2 \mathrm{~T} 5}$ in the dpp domain) (C) reduces expression of hsp23, whereas an increase in its activity (overexpression of UAS- $\mathrm{N}_{\mathrm{i} 12.1}$ ) increases levels of hsp23 in all the imaginal discs (D). Lack of EGFR signaling (overexpression of UAS-DN Raf $_{3.1}$ in dpp domains) (E), or its increase (overexpression of UAS-Ras $\mathrm{V}_{\mathrm{V} 2}$ in dpp domains) (F), alters the expression of hsp23 in a context-dependent manner. Arrowheads identify signaling territories with ectopic or high levels of hsp23 expression, and arrows represent territories with lower levels of hsp23 expression than in the wild-type. probably by promoting expression of AS-C genes (Fig. 6F). Together, these results show that hsp23 expression might be involved in proneural differentiation and is regulated by mechanisms independent of heat shock and specific to each imaginal disc.

\section{Discussion}

This study is part of an effort of our laboratory to monitor changes in polypeptide synthesis involved in cell proliferation and differentiation in Drosophila. We use the wing imaginal discs both as a model system and as a quantitative protein database that can be used for comparative purposes in genetic studies. Here, we focused on the purification of a polypeptide (catalogued as SSP 6002) that showed different levels of expression in different imaginal discs. Preparative 2-D gels followed by MALDI-TOF analysis revealed that polypeptide SSP 6002 is heat shock protein 23. We confirmed this conclusion by showing that its expression was elevated six-fold $2 \mathrm{~h}$ after heat shock. The polypeptide is expressed constitutively in a single unphosphorylated form, as revealed by Western blotting with an antibody against the denaturated protein.

Hsp23 attracted attention some time ago, when it was found that tissues from late third instar larvae had much higher levels of this polypeptide than tissues from mid-third instar larvae [40]. This finding led to the suggestion that hsp23 has a function in normal development as well as in the heat shock response. Over the years, evidence that shsps in general, and hsp23 in particular, are involved in processes other than merely heat shock responses has increased, and in fact we encountered this protein during a comparative 
study of levels of protein expression in different imaginal discs. Our observations agree with the distinctive tissue-specific and age-dependent expression of hsp23 reported by others, and suggests that this protein may have different functions in different Drosophila organs.

We found that expression of hsp23 in imaginal discs from the early third instar is restricted to territories coincident with neural differentiation (proneural clusters). During the third instar larva and early pupal stages, sensory organ mother cells (SMCs) appear in precise positions in the imaginal discs, enabling macrochaetae to emerge at defined positions [41]. All cells in the proneural clusters, including the SMCs (marked by the expression of neuralized), expressed hsp23 throughout imaginal development. Interestingly, the development of sensory organs in D. melanogaster and D. simulans is controlled by changes in the level of expression of the AC-SC genes associated with differences in the temperature of development [42], strongly suggesting that hsp23 expression is correlated with proneural specification.

Another striking conclusion from our analysis is that the restriction of hsp23 to proneural clusters is independent of temperature. Thus, although heat shock increases the quantity of the protein, it does not alter its pattern of expression. This finding challenges the traditional definition of a heat shock protein and raises the question of whether other hsps are induced by heat shock in those cells in which hsp23 seems not to be expressed.

In contrast to heat shock induction, changes in the expression or activity of transcription factors $(s c)$ and signaling pathways ( $\mathrm{N}$ and EGFR) involved in proneural specification did modify the hsp23 pattern. Thus, the high and autonomous level of hsp23 expression in imaginal discs when sc is overexpressed demonstrates the ability of proneural genes to regulate its expression. Nevertheless, unlike AS-C genes expression, which is progressively restricted to SMCs, expression of hsp23 continued during sensory specification and differentiation (from third instar larva to early pupal stages) in the group of cells that constitute the proneural clusters. This result shows that, in addition to its control by the AS-C genes, expression of hsp23 is dependent on the signaling pathways required to specify proneural cluster. Two major candidates are $\mathrm{N}$ and EGFR, whose antagonistic activity maintains the integrity and dimensions of proneural clusters needed to specify SMCs after proneural cluster formation.

We have shown that signaling pathways influence hsp23 expression in a context-dependent manner, indicating that there are specific systems of regulation for the different imaginal discs. We suggest that the pattern of hsp23 expression is dependent on specific signaling pathways and transcription factors during development, and further that it is controlled by selector genes such as Ubx. We further propose that expression in wing discs is higher than in the haltere in the wild-type condition, as well as in a Ubx-defective genetic background, because it is up-regulated by higher levels of $s c$ expression. This hypothesis is supported by the repression of sc exercised by Ubx in the haltere [43] and the capacity of cells expressing $s c$ ectopically to induce ectopic expression of hsp23 in the haltere pouch.

Apart from its restricted expression during imaginal development, hsp23 has a dynamic pattern during the pupal stages that is not associated with neural territories. This finding raises new questions about the function of hsp23 in pupal development and its mechanism of regulation.

In summary, we have shown that hsp23, and probably, by extension, other heat shock proteins, may perform important functions during development. In addition, their expression appears to require precise mechanisms of regulation independent of heat shock.

$J$.A. is the recipient of a predoctoral fellowship of the Universidad Autónoma de Madrid. L.A.B-L is a fellow student of Consejo Superior de Investigaciones Científicas in collaboration with PACISA-GIRALT (I3P-BPD2002-1). This work was supported by Grant PB87-0449 from Comision Asesora de Investigación Científica y Técnica and by an institutional grant from Fundación Ramón Areces.

\section{References}

[1] Santarén, J. F., Assiego, R., García-Bellido, A., Roux's Arch. Dev. Biol. 1993, 203, 131-139.

[2] Santarén, J. F., García-Bellido, A., Dev. Genes Evol. 1996, 206, 349-354.

[3] Sirotkin, K., Davidson, N., Dev. Biol. 1982, 89, 196-210.

[4] Mason, P. J., Hall, L. M. C., Gauss, J., J. Mol. Gen. Genet. 1984, 194, 73-78.

[5] Pauli, D., Arrigo, A. P., Vazquez, J., Tonka, C. H., Tissières, A., Genome 1989, 31, 671-676.

[6] Southgate, R., Ayme, A., Voellmy, R., J. Mol. Biol. 1983, 165, 35-37.

[7] Marin, R., Tanguay, R. M., Chromosoma 1996, 105, 142-149.

[8] Lavoie, J. N., Gingras-Breton, G., Tanguay, R. M., Landry, J., J. Biol. Chem. 1993, 268, 3420-3429.

[9] Bruey, J. M., Ducasse, C., Bonniaud, P., Ravagnan, L., Susin, S. A., Diaz-Latoud, C., Gurbuxani, S. et al., Nat. Cell Biol., 2000, 2, 645-652.

[10] Arrigo, A. P., Fakan, S., Tissières, A., Devel. Biol. 1980, 78, 86103.

[11] Ding, L., Candido, E. P., J. Biol. Chem. 2000, 275, 9510-9517.

[12] Michaud, S., Marin, R., Westwood, J. T., Tanguay, R. M., J. Cell Sci. 1997, 110, 1989-1997.

[13] Lang, L., Miskovic, D., Fernando, P., Heikkila, J. J., Dev. Genet. 1999, 25, 365-374.

[14] Armstrong, C. L., Krueger-Naug, A.M., Currie, R. W., Hawkes, R., J. Comp. Neurol. 2001, 434, 262-274.

[15] Jantschitsch, C., Kindas-Mugge, I., Metze, D., Amann, G., Micksche, M., Trautinger, F., Br. J. Dermatol. 1998, 139, 247253. 
[16] Glaser, R. L., Wolfner, M. F., Lis, J. T., EMBO J. 1986, 5, 747754.

[17] Ayme, A., Tissières, A., EMBO J. 1985, 4, 2949-2954.

[18] Glaser, R. L., Lis, J. T., Mol. Cell Biol. 1990, 10, 131-137.

[19] Marin, R., Valet, J. P., Tanguay, R., Dev. Gen. 1993, 14, 69-77.

[20] Haass, C., Klein, U., Kloetzel, P. M., J. Cell Sci. 1990, 96, 413418.

[21] Egger, B., Leemans, R., Loop, T., Kammermeier, L., Fan, Y., Radimerski, T., Strahm, M. C. et al., Development 2002, 129, 3295-3309.

[22] Michaud, S., Tanguay, R. M., BMC Dev. Biol. 2003, 3, 9.

[23] Calleja, M., Moreno, E., Pelaz, S., Morata, G., Science 1996, 111, 252-255.

[24] Wilder, E. L., Perrimon, N., Development 1995, 121, 477-488.

[25] Parras, C., Garcia-Alonso, L. A., Rodriguez, I., Jimenez, F., EMBO J. 1996, 15, 6394-6399.

[26] Seugnet, L., Simpson, P., Haenlin, M., Development 1997, 124, 2015-2025.

[27] Klein, T., Brennan, K., Arias, A. M., Dev. Biol.1997, 189, 123134.

[28] Karim, F. D., Rubin, G. M., Development 1998, 125, 1-9.

[29] Martin-Blanco, E., Roch, F., Noll, E., Baonza, A., Duffy, J. B., Perrimon, N., Development 1999, 126, 5739-5747.
[30] Huang, F., Dambly-Chaudiere, C., Ghysen, A., Development 1991, 111, 1087-1095.

[31] Santarén, J. F., Van Damme, J., Puype, M., Vandekerckhove, J., García-Bellido, A., Exp. Cell Res. 1993, 206, 220-226.

[32] Santarén, J. F., Electrophoresis 1990, 11, 254-267.

[33] O'Farrell, P. Z., J. Biol. Chem. 1975, 250, 4007-4021.

[34] Laskey, R. A., Mills, A. D., Eur. J. Biochem. 1975, 56, 335-341.

[35] Alonso, J., Santarén, J. F., Proteomics 2005, 5, 474-489.

[36] Gisselbrecht, S., Skeath, J. B., Doe, C. Q., Michelson, A. M., Genes Dev. 1996, 10, 3003-3017.

[37] Baena-López, L. A., García-Bellido, A., Development 2003, 130, 197-208.

[38] Peifer, M., Karch, F., Bender, W., Genes Dev. 1987, 1, 891-898.

[39] Culi, J., Martín-Blanco, E., Modolell, J., Development 2001, 128, 299-308.

[40] Cheney, C. M., Shearn, A., Dev. Biol. 1983, 95, 325-330.

[41] Cubas, P., de Celis, J. F., Campuzano, S., Modolell, J., Genes Dev. 1991, 5, 996-1008.

[42] Skaer, N., Simpson, P., Dev. Biol. 2000, 221, 148-167.

[43] Weatherbee, S. D., Halder, G., Kim, J., Hudson, A., Carroll, S., Genes Dev. 1998, 12, 1474-1482. 